\title{
Excitation and reception of pure shear horizontal waves by using face-shear $\mathrm{d}_{24}$ mode piezoelectric wafers
}

\author{
Hongchen Miao ${ }^{1,2}$, Qiang Huan ${ }^{1}$, Faxin $\mathrm{Li}^{1,2, \mathrm{a})}$ \\ ${ }^{1}$ LTCS and Department of Mechanics and Engineering Science, College of Engineering, Peking \\ University, Beijing, 100871, China \\ ${ }^{2}$ Center for Applied Physics and Technology, Peking University, Beijing, 100871, China
}

\begin{abstract}
The fundamental shear horizontal ( $\mathrm{SHO}$ ) wave in plate-like structures is of great importance in non-destructive testing (NDT) and structural health monitoring (SHM) as it is non-dispersive, while excitation and reception of SH0 waves using piezoelectrics is always a challenge. In this work, we firstly demonstrate via finite element simulations that face-shear piezoelectrics is superior to thickness-shear piezoelectrics in driving SH waves. Next, by using a newly defined face-shear $d_{24}$ PZT wafer as actuator and face-shear $d_{36}$ PMN-PT wafers as sensors, pure SH0 wave was successfully excited in an aluminum plate from $140 \mathrm{kHz}$ to $190 \mathrm{kHz}$. Then, it was shown that the face-shear $\mathrm{d}_{24}$ PZT wafer could receive the SH0 wave only and filter the Lamb waves over a wide frequency range $(120 \mathrm{kHz}$ to $230 \mathrm{kHz})$. The directionality of the excited SH0 wave was also investigated using face-shear $\mathrm{d}_{24}$ PZT wafers as both actuators and sensors. Results show that pure SH0 wave can be excited symmetrically along two orthogonal directions $\left(0^{\circ}\right.$ and $90^{\circ}$ ) and the amplitude of the excited SH0 wave can keep over $90 \%$ of the maximum amplitude when the deviate angle is within $30^{\circ}$. This work could greatly promote the applications of $\mathrm{SH}$ waves in NDT and SHM.
\end{abstract}

Keywords: shear horizontal waves; guided waves; face-shear piezoelectrics; non-destructive testing (NDT), structural health monitoring (SHM).

\footnotetext{
a) Author to whom all correspondence should be addressed, Email: lifaxin@pku.edu.cn
} 


\section{Introduction}

Ultrasonic guided wave inspection is a very efficient method in non-destructive testing (NDT) and structural health monitoring (SHM)[1]. The fundamental shear horizontal (SH0) waves in plate-like structures and torsional $[\mathrm{T}(0,1)]$ waves in pipes are especially useful for long-distance NDT due to their non-dispersive characteristics thus simplifying the interpretation of signals [2, 3]. Furthermore, as there is no out-of-plane particle displacement in SH0 wave and T(0,1) wave, they will be less affected by the presence of surrounding media. In addition, there is no mode conversion when these waves travel across defects or boundaries, e.g., SH0 wave may change to higher mode SH waves but will not convert to Lamb waves when encountering defects. Actually, the SH0 wave in plates is essentially equivalent to the $\mathrm{T}(0,1)$ wave in a thin pipe-like structure[4], so in most cases the conclusions obtained from one can be extended to the other straightforwardly.

Currently, although several methods have been proposed to generate SH0 or T(0,1) waves, it is still very difficult to obtain pure SH0 wave or $\mathrm{T}(0,1)$ wave mode efficiently. The well-known electromagnetic acoustic transducer (EMAT) can generate pure SH0 wave mode[5, 6], but EMATs can only work on conductive materials and the signal-to-noise ratio (SNR) is usually not high because of weak coupling with the structures due to its non-contact. Liu et al. employed large $(12.5 \mathrm{~mm} \times 8 \mathrm{~mm} \times 4.5 \mathrm{~mm})$ thickness-shear $\left(\mathrm{d}_{15}\right)$ mode piezoelectric transducers to generate $\mathrm{T}(0,1)$ wave in pipes[7]. Recently, Kamal et al. used thickness-shear $\left(\mathrm{d}_{15}\right)$ mode piezoelectric wafers to generate SH0 wave in plates perpendicular to the poling direction, but strong lamb waves will be excited simultaneously along the poling direction[8]. Later, Boivin et al. showed that the amplitude of the co-excited Lamb waves can be decreased by optimizing the geometry of the $d_{15}$ mode piezoelectric wafer at a given frequency[9]. It should be noted that the energy conversion efficiency of thickness-shear $\left(\mathrm{d}_{15}\right)$ mode piezoelectric transducers is fairly low and the amplitude of the obtained SH0 wave is usually very small[8]. Zhou et al. used a face-shear $d_{36}$ mode PMN-PT crystal transducer to excite and receive SH0 waves in plates[10, 11]. Similarly, Lamb waves were also excited, since the extensional $d_{31}$ mode co-existed with the face-shear $d_{36}$ mode in PMN-PT crystals[12]. Moreover, the low Curie temperature and high cost of PMN-PT crystal make it not suitable for applications in NDT or SHM. Recently, we realized the face-shear $d_{36}$ mode in $\mathrm{PbZr}_{1-\mathrm{x}} \mathrm{Ti}_{\mathrm{x}} \mathrm{O}_{3}$ (PZT) ceramics and successfully excited $\mathrm{SH} 0$ wave in an aluminum plate 
using $d_{36}$ mode PZT wafers[13-15]. Since the extensional $d_{31}$ mode also existed in the $d_{36}$ mode PZT ceramics, pure SH0 wave still cannot be obtained[15].

In this work, a newly defined face-shear $\mathrm{d}_{24}$ PZT wafer (in-plane poled and electric field applied along another orthogonal in-plane direction) was proposed to excite and receive pure SH0 wave. Firstly, we demonstrate via finite element simulations that face-shear piezoelectric transducers are superior to thickness-shear ones in exciting SH waves in plates. Then we successfully excited pure SH0 waves and received the SH0 wave only in an aluminum plate using the face-shear $\mathrm{d}_{24}$ mode PZT wafers as actuators or sensors. Finally, the directionality of the excited SH0 wave was investigated using the $\mathrm{d}_{24}$ mode PZT wafers as both actuators and sensors.

\section{Methodology}

The first step in developing a SH wave transducer is to understand the excitation mechanism. It is well known that there exist two different families of guided waves in plates, i.e., Lamb waves and shear horizontal $(\mathrm{SH})$ waves. The particle vibration caused by all the $\mathrm{SH}$ modes are in a plane parallel to the plate surface, and SH waves propagate perpendicular to the direction of particle's vibration[1]. Therefore, the thickness-shear $d_{15}$ mode transducer is a straightforward solution, since it may cause particle vibration in the $\mathrm{x}$ direction and $\mathrm{SH}$ waves can be excited in the $\mathrm{z}$ direction, as shown in Fig. 1(a). However, the energy conversion efficiency of the thickness-shear $d_{15}$ transducer is very low. Fig. 1(b) shows the finite element (FEM) simulated deformation of a thickness-shear $d_{15}$ PZT-5H wafer with dimensions of $6 \mathrm{~mm} \times 6 \mathrm{~mm} \times 1 \mathrm{~mm}$ bonded on a 1 mm-thick aluminum plate through a thin bond layer. The thickness of the bond layer is set as $\mathbf{5 0}$ $\mu \mathrm{m}$ and its elastic modulus is $1 \mathrm{GPa}$. It can be seen that when bonded on an aluminum plate, the deformation of the thickness-shear $d_{15}$ wafer under a dc voltage $(20 \mathrm{~V})$ trends to be the simple shear deformation. i.e., the displacement of the bottom surface of the transducer is nearly zero and almost no deformation was transformed to the aluminum plate. Therefore, the SH waves in the plate can only be caused by the inertia force and the non-linear deformation of the thickness-shear $d_{15}$ piezoelectric wafer under AC voltages, resulting in a relative low efficiency of energy transfer.

Alternatively, inducing face-shear deformation in plates is another solution to generate $\mathrm{SH}$ waves, 
which is the excitation mechanism of the $\mathrm{d}_{36}$ mode piezoelectric transducers (crystals or ceramics). As mentioned above, the field induced deformation of the $\mathrm{d}_{36}$ mode piezoelectric transducers is always the superposition of an extensional deformation and a pure face-shear deformation, since the extensional $d_{31}$ mode co-existed with the $d_{36}$ mode in $d_{36}$ type piezoelectric crystals and ceramics[12, 13]. Therefore, it is very difficult to excite pure $\mathrm{SH}$ wave by using the $\mathrm{d}_{36}$ type piezoelectric transducers[11, 15]. Here a newly defined face-shear $\mathrm{d}_{24}$ mode piezoelectric transducer was proposed to induce pure face-shear deformation in plates. As shown in Fig. 1(c), the PZT based face-shear $\mathrm{d}_{24}$ wafer was in-plane poled along the " 3 " direction. The driving field is applied along another in-plane direction, i.e., the "2" direction and the thickness is along the "1" direction. Pure face-shear deformation is expected to be obtained in this type transducer under applied voltage, since $d_{24}$ mode is the unique deformation mode when the field is applied along the "2" direction. Compared with the conventional thickness-shear $d_{15}$ PZT transducer, the proposed face-shear $\mathrm{d}_{24}$ PZT transducer is more efficient in driving SH waves in plates. Fig. 1(d) shows the FEM simulated deformation of a face-shear $\mathrm{d}_{24}$ wafer $(6 \mathrm{~mm} \times 6 \mathrm{~mm} \times 1 \mathrm{~mm})$ bonded on a $1 \mathrm{~mm}$-thick aluminum plate under a dc voltage of $20 \mathrm{~V}$. It can be seen that the face-shear deformation of the transducer can be effectively transferred to the plate through the bonding layer (the in-plane displacement transfer ratio is over 60\%). The face-shear deformation will then cause the traction force $\tau_{x z}$ in the plate and the SH waves will be launched in both $\mathrm{z}$ direction and $\mathrm{x}$ direction, as indicated in Fig. 1(c). 
(a)

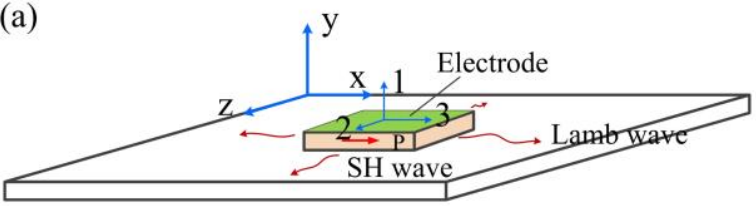

Thickness-shear $d_{15}$ PZT transducer

(c)

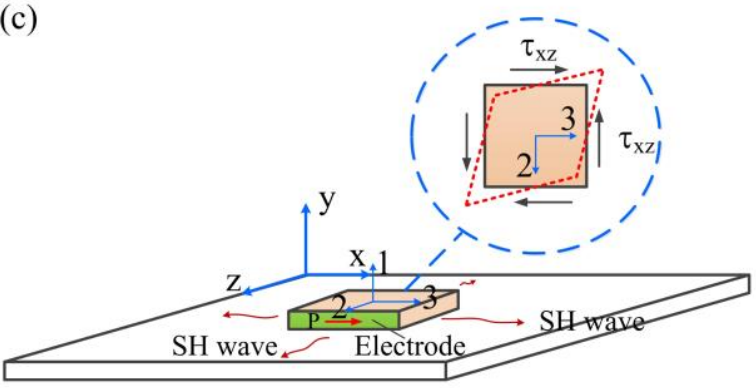

Face-shear $\mathrm{d}_{24}$ PZT transducer (b)

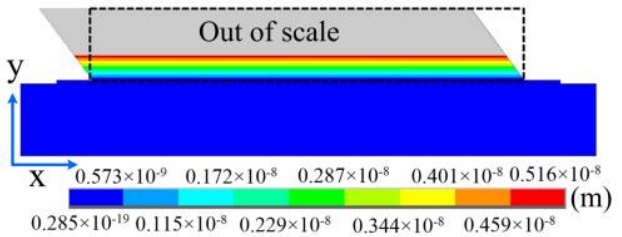

(d)

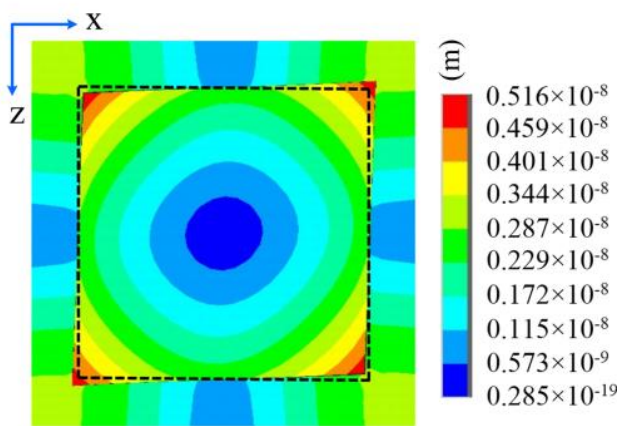

Fig. 1 Schematics of (a) the conventional thickness-shear $d_{15}$ PZT transducer and (c) the face-shear $\mathrm{d}_{24}$ PZT transducer boned on a plate. FEM simulated in-plane deformations of (a) and (c) are shown in (b) and (d), respectively.

Experiments were then conducted to explore the face-shear $\mathrm{d}_{24}$ PZT transducer's performance on excitation and reception of SHO wave. An aluminum plate with the dimensions of $1000 \mathrm{~mm} \times 1000 \mathrm{~mm} \times 1 \mathrm{~mm}$ was used in this study. The commercially available PZT-5H piezoelectric ceramics were used to fabricate the face-shear $d_{24}$ PZT wafer with dimensions of $6 \mathrm{~mm} \times 6 \mathrm{~mm} \times 1.5 \mathrm{~mm}$. The $\mathrm{d}_{24}$ wafer exhibits good face-shear performance from $130 \mathrm{kHz}$ to $230 \mathrm{kHz}$, which was confirmed by its impedance spectrum as shown in Fig. 2(a). In the wave excitation/reception testing, firstly the face-shear $d_{24}$ PZT wafer served as the actuator to excite SH0 wave. A d $\mathrm{d}_{36}$ type PMN-PT crystal patch $\left(\mathrm{d}_{36}=1600 \mathrm{pC} / \mathrm{N}\right.$ and $\left.\mathrm{d}_{31}=-360 \mathrm{pC} / \mathrm{N}\right)$ with dimensions of $5 \mathrm{~mm} \times 5 \mathrm{~mm} \times 1 \mathrm{~mm}$ was used as the sensor to check the purity of the excited SH wave, since it can receive both $\mathrm{SH}$ wave and Lamb waves[11]. Meanwhile, a $\mathrm{d}_{31}$ type PZT-5H sensor ( $6 \mathrm{~mm} \times 6 \mathrm{~mm} \times 1 \mathrm{~mm}$ ), which can only receive Lamb waves, was also used to detect the possible A0 and S0 waves. Then, to check whether the $\mathrm{d}_{24}$ PZT transducer could only receive $\mathrm{SH}$ wave, a $\mathrm{d}_{36}$ type PZT-4 wafer $(6.3 \mathrm{~mm} \times 6.3 \mathrm{~mm} \times 1 \mathrm{~mm})$ which can generate both $\mathrm{SH}$ waves and Lamb waves, was used as the actuator. Detailed properties of the $d_{36}$ type PZT- 4 patch can be found in our recent work [15]. Besides the $d_{24}$ PZT wafer, a $d_{36}$ type PMN-PT crystal wafer ( $5 \mathrm{~mm} \times 5 \mathrm{~mm} \times 1 \mathrm{~mm}$ ) was also used as sensor for comparison. Finally, the face-shear $\mathrm{d}_{24}$ PZT 
wafers were used as both actuator and sensors to check the directionality of the excited SH0 wave. The layout and location of the actuator and sensors were shown in Fig. 2(b). All the actuators were driven by using a function generator (33220A, Agilent, USA), into which a five-cycle Hanning window-modulated sinusoid toneburst was preprogrammed. A power amplifier (KH7602M) was used to amplify the drive signal and an Agilent DSO-X 3024A oscilloscope was used to collect the wave signals received by sensors.
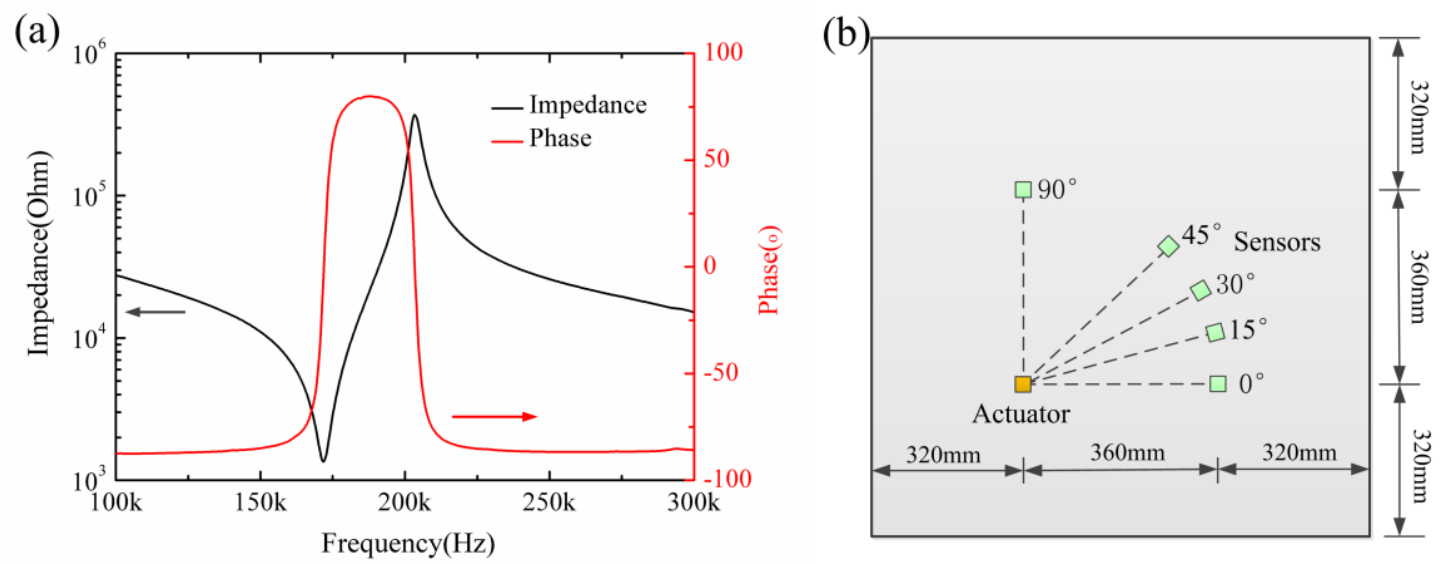

Fig. 2 (a) The impedance spectrum of the free face-shear $d_{24}$ PZT wafer with dimensions of $6 \mathrm{~mm} \times 6 \mathrm{~mm} \times 1.5 \mathrm{~mm}$. (b) Schematic of the layout of the piezoelectric actuator and sensors for wave excitation and reception in an aluminum plate.

\section{Results and Discussions}

Fig. 3(a) shows the wave signals excited by the face-shear $d_{24}$ PZT actuator and received by a $d_{36}$ type PMN-PT sensor placed on $0^{\circ}$ direction. The central frequency of the drive signal was first fixed at $150 \mathrm{kHz}$ and its amplitude was set to be $20 \mathrm{~V}$. It can be seen from Fig. 3(a) that only SH0 wave with high signal-to-noise ratio (SNR) was detected and no other unexpected wave modes (such as S0 or A0 wave mode) were observed. As expected, the waveform of the received SH0 wave is almost the same as that of the incident pulse signal, i.e., no dispersion was observed. By using continuous wavelet transform (CWT) to analyze these signals, we can extract the time interval of $120.56 \mu$ s between the actuator and the sensor with the distance of $360 \mathrm{~mm}$, as shown in Fig. 3(b). The calculated group velocity of $2986 \mathrm{~m} / \mathrm{s}$ is in good agreement with the theoretical group velocity $(3099 \mathrm{~m} / \mathrm{s})$ of SH0 wave in the aluminum plate. Moreover, the vanishing signals 
received by the $\mathrm{d}_{31}$ type PZT sensor indicates that no $\mathrm{S} 0$ wave or $\mathrm{A} 0$ wave were generated, as shown in Fig. 3(c). Therefore, the testing results in Fig. 3 had verified that the proposed face-shear $\mathrm{d}_{24}$ PZT wafer can excite pure SH0 wave at $150 \mathrm{kHz}$.
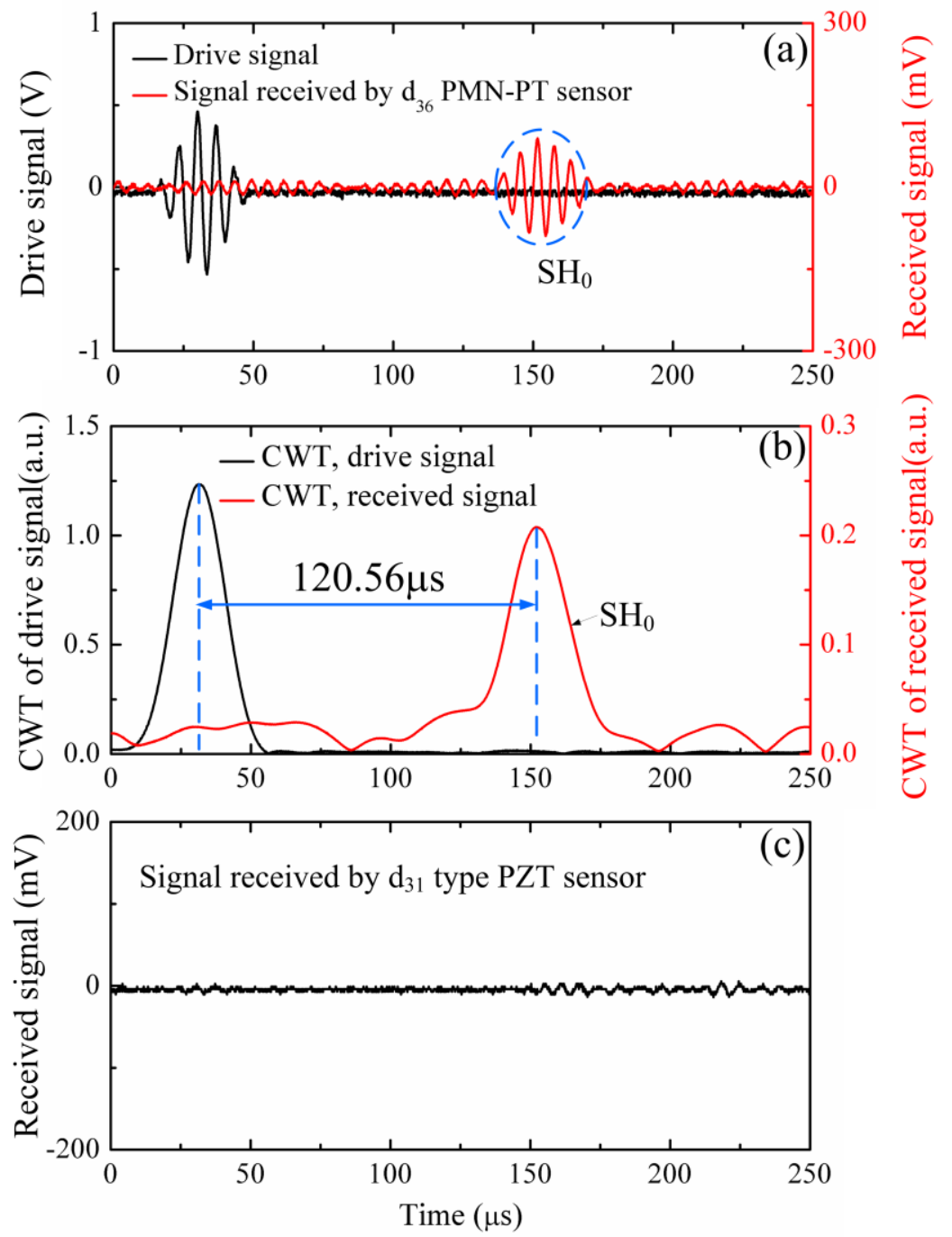

Fig. 3 Driving wave signals applied to the face-shear $\mathrm{d}_{24}$ PZT actuator at $150 \mathrm{kHz}$ and received signals by (a) a $\mathrm{d}_{36}$ type PMN-PT crystal sensor and (c) a $\mathrm{d}_{31}$ type PZT sensor placed at $0^{\circ}$ direction. (b) Continuous wavelet transform (CWT) of the driving and received wave signals shown in Fig. 3(a).

Fig. 4 shows the wave signals excited by the face-shear $d_{24}$ PZT actuator at different central frequencies and received by a $\mathrm{d}_{36}$ type PMN-PT sensor placed on $0^{\circ}$ direction. It can be seen that pure SH0 wave with high SNR can be excited by the face-shear $d_{24}$ PZT wafer over a wide frequency range ( $140 \mathrm{kHz}$ to $190 \mathrm{kHz}$ ). Fig. 4(a) shows that pure SH0 wave can also be excited by 
the $\mathrm{d}_{24}$ wafer at $130 \mathrm{kHz}$. However, the amplitude of the excited SH0 wave is relative small, since the exciting frequency is far away from the resonance frequency of the $\mathrm{d}_{24}$ mode $(192 \mathrm{kHz}$ for the bonded $\mathrm{d}_{24}$ PZT wafer). When the exciting frequency was increased to $200 \mathrm{kHz}, \mathrm{Fig}$. $4(\mathrm{~g})$ shows that the S0 wave was also excited, which was further confirmed by wave signals detected by a $\mathrm{d}_{31}$ PZT sensor, as shown in Fig. 4(h). Note that the frequency range from $140 \mathrm{kHz}$ to $190 \mathrm{kHz}$ is of practical significance, since frequencies in the range of $10 \mathrm{kHz}$ to $250 \mathrm{kHz}$ are often used in engineering [16-18]. Also as expected, the waveform and group velocity of the excited SH0 wave are frequency-independent. On the other hand, it can be seen from Fig. 4 that the amplitude of the excited SH0 wave increases steadily with the increasing drive frequency, which may attribute to the fact that the face-shear deformation of the $\mathrm{d}_{24}$ PZT wafer was amplified near the resonance frequency and the size of the $\mathrm{d}_{24}$ PZT wafer became more and more close to the half of wavelength with the increasing drive frequency.
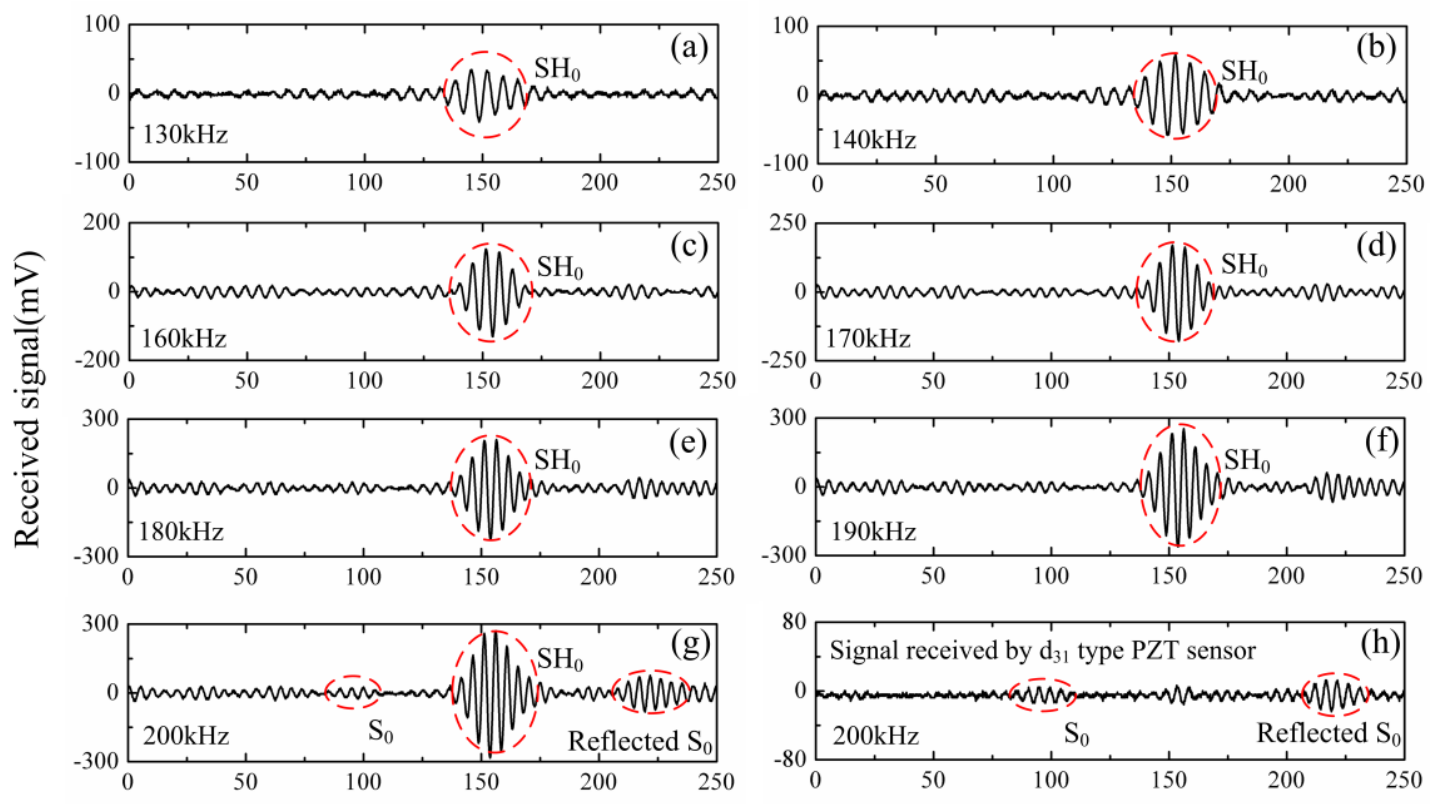

Time $(\mu \mathrm{s})$

Fig. 4 Wave signals excited by the face-shear $d_{24}$ PZT actuator at different frequencies and received by a $\mathrm{d}_{36}$ type PMN-PT crystal sensor ((a)-(g)) placed at $0^{\circ}$ direction. (h) Wave signals excited by the $\mathrm{d}_{24}$ PZT actuator at $200 \mathrm{kHz}$ and received by a $\mathrm{d}_{31}$ PZT sensor placed at $0^{\circ}$ direction.

Then the face-shear $d_{24}$ PZT wafer served as a sensor to check its performance on receiving the 
SH0 wave. Here a $\mathrm{d}_{36}$ type PZT-4 wafer was used as the actuator to excite both SH and Lamb waves. For comparison, a $\mathrm{d}_{36}$ type PMN-PT crystal was also used as the sensor to receive the excited waves. It can be seen from Fig. 5(a) that both SH0 wave and A0 wave were successfully excited by using the $\mathrm{d}_{36}$ type PZT-4 wafer under a voltage of $40 \mathrm{~V}$ at $120 \mathrm{kHz}$. However, when the excited waves were received by the $\mathrm{d}_{24}$ PZT sensor, only SH0 wave was detected, as shown in Fig. 5(b). When the driving frequency varies from $120 \mathrm{kHz}$ to $170 \mathrm{kHz}$, the same phenomenon was observed and here only the results at $170 \mathrm{kHz}$ were given, as shown in Fig. 5(c) and (d). In addition, when the central frequency of the excitation signal increased from $170 \mathrm{kHz}$ to $230 \mathrm{kHz}$, both lamb waves (A0 and/or S0 wave) and SH0 wave were excited simultaneously by the $\mathrm{d}_{36}$ type PZT-4 actuator, and all these wave modes can be detected by the $\mathrm{d}_{36}$ PMN-PT sensor. Again, when these waves were detected by the $\mathrm{d}_{24}$ PZT sensor, only the SH0 wave was received and the lamb waves (A0 or S0 wave) were excluded. For simplicity, only the results at $220 \mathrm{kHz}$ were given, as shown in Fig. 5(e) and (f). However, when the driving frequency increased to $240 \mathrm{kHz}$, Fig. (h) shows that the $d_{24}$ PZT sensor cannot filter the S0 wave reflected from the plate boundary. All these experiments indicate that the face-shear $\mathrm{d}_{24}$ PZT wafer can also be used as a selective sensor, which can only receive SH wave and exclude the Lamb waves over a wide frequency range (120 $\mathrm{kHz}$ to $230 \mathrm{kHz}$ in this work). Moreover, the wave form of the SH0 wave received by the $\mathrm{d}_{24} \mathrm{PZT}$ sensor is more perfect than that received by the $d_{36}$ type PMN-PT sensor, as shown in Fig. 5. To the author's knowledge, so far no sensor has been reported that can only receive the $\mathrm{SH}$ wave and exclude the lamb waves in a wide frequency range. 

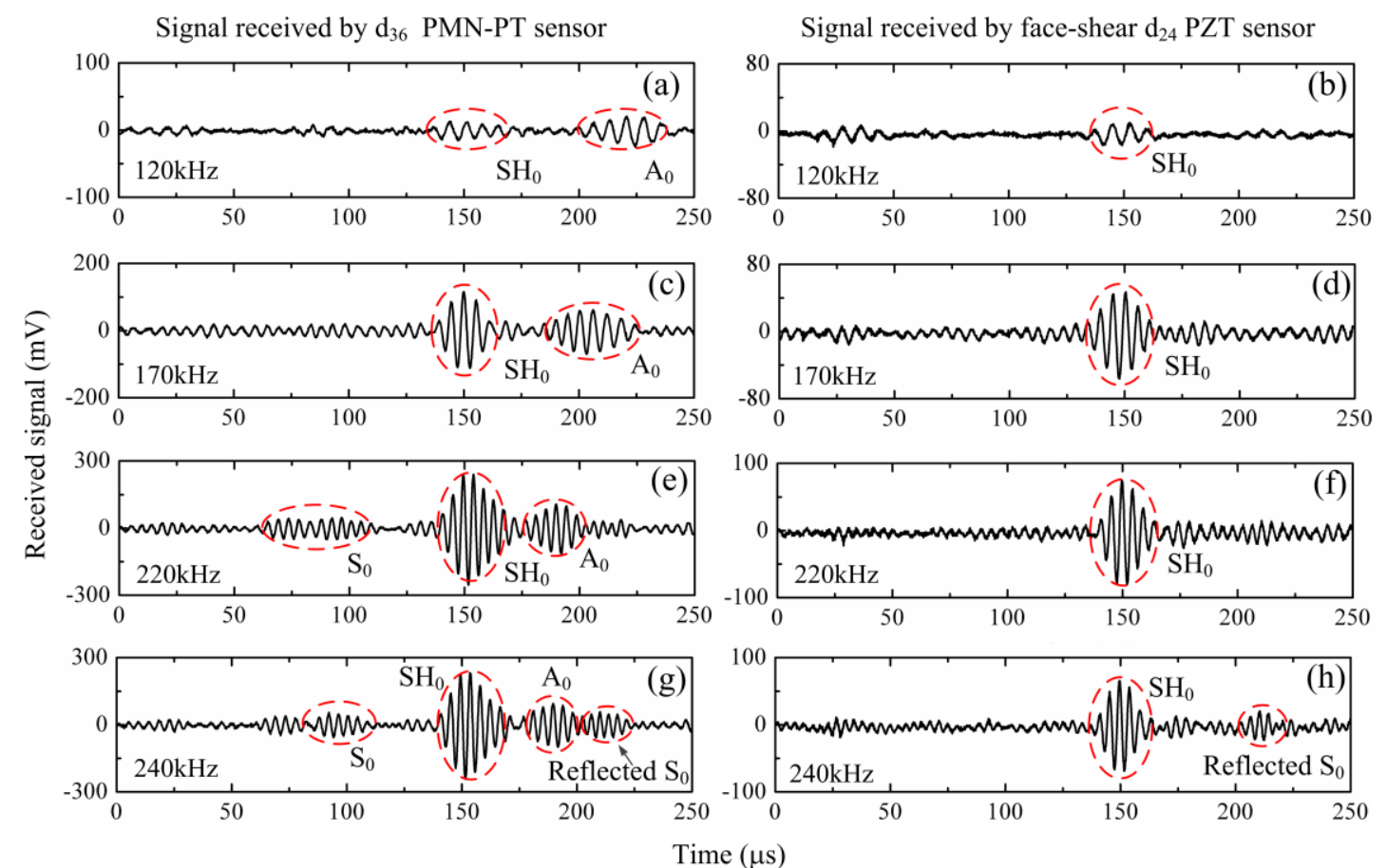

Fig. 5 Wave signals excited by a $\mathrm{d}_{36}$ type PZT- 4 actuator at different frequencies and received by a $\mathrm{d}_{36}$ type PMN-PT sensor (left column) and by the face-shear $\mathrm{d}_{24}$ PZT sensor (right column) placed at $0^{\circ}$ direction.

The directionality of the SH0 wave excited by the $\mathrm{d}_{24}$ PZT transducer was also investigated. Fig. 6(a) and (b) show the FEM simulated displacement wavefields excited by the $d_{24}$ PZT transducer bonded on a $1 \mathrm{~mm}$-thick aluminum plate under a voltage of $20 \mathrm{~V}$ at $150 \mathrm{kHz}$. As we know, the $\mathrm{SH}$ waves propagate perpendicular to the direction of particle vibration, so the tangential displacement component in the cylindrical coordinate represents the SH0 wave, as shown in Fig. 6(a) . It can be seen that the SH0 wave was excited along two orthogonal directions $\left(0^{\circ}\right.$ and $\left.90^{\circ}\right)$ which can be defined as the main directions. Moreover, Fig. 6(a) shows that the excited SH0 wave is symmetric along the $45^{\circ}$ direction. As indicted in our recent work[15], the pure face-shear deformation $\left(S_{6}\right)$ is equivalent to the superposition of an elongation (or contraction) deformation along the $45^{\circ}$ direction $\left(S_{45^{\circ}}\right)$ and a contraction (elongation) deformation along the $135^{\circ}$ direction $\left(S_{135^{\circ}}\right)$. Therefore, theoretically the Lamb waves could be excited along the $45^{\circ}$ (or $135^{\circ}$ ) direction. The FEM simulated results show that the amplitude of Lamb waves along $45^{\circ}$ (or $135^{\circ}$ ) direction is quite small, since the tangential displacement component occupies about $95 \%$ of the total 
displacement, as shown in Fig. 6(a) and (b).

Experiments were then conducted to examine the FEM simulated results. Fig. 6(c) shows the SH0 wave excited and received by the face-shear $\mathrm{d}_{24}$ PZT wafer along different propagation directions. The central frequency of the drive signal was fixed at $150 \mathrm{kHz}$ and its amplitude was set to be 20 V. As expected, pure SH0 wave were excited along two orthogonal main directions $\left(0^{\circ}\right.$ and $\left.90^{\circ}\right)$, as shown in Fig. 6(c). Furthermore, when the deviate angle referring to the main direction $\left(0^{\circ}\right.$ or $90^{\circ}$ ) is less than $30^{\circ}$, no pronounced amplitude change of the SH0 wave was observed. At $45^{\circ}$ direction, almost no wave can be detected by the face-shear $\mathrm{d}_{24}$ PZT sensor. A $\mathrm{d}_{31}$ type PZT sensor was further placed at the $45^{\circ}$ direction to detect the possible Lamb waves and the result was plotted in the last subfigure of Fig. 6(c). It can be seen that S0 and A0 wave modes were indeed detected along the $45^{\circ}$ direction, but their amplitude is rather small and can be neglected.

(a)

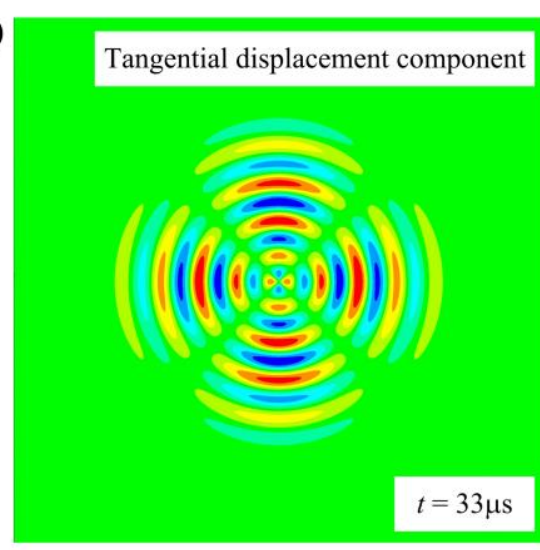

$0.461 \mathrm{~nm}$

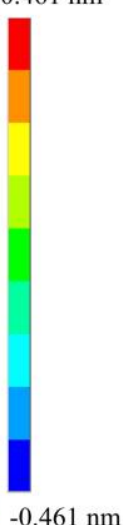

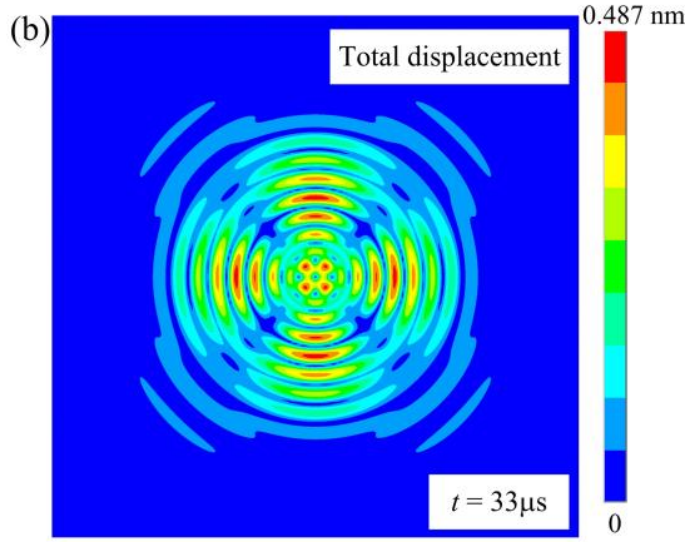
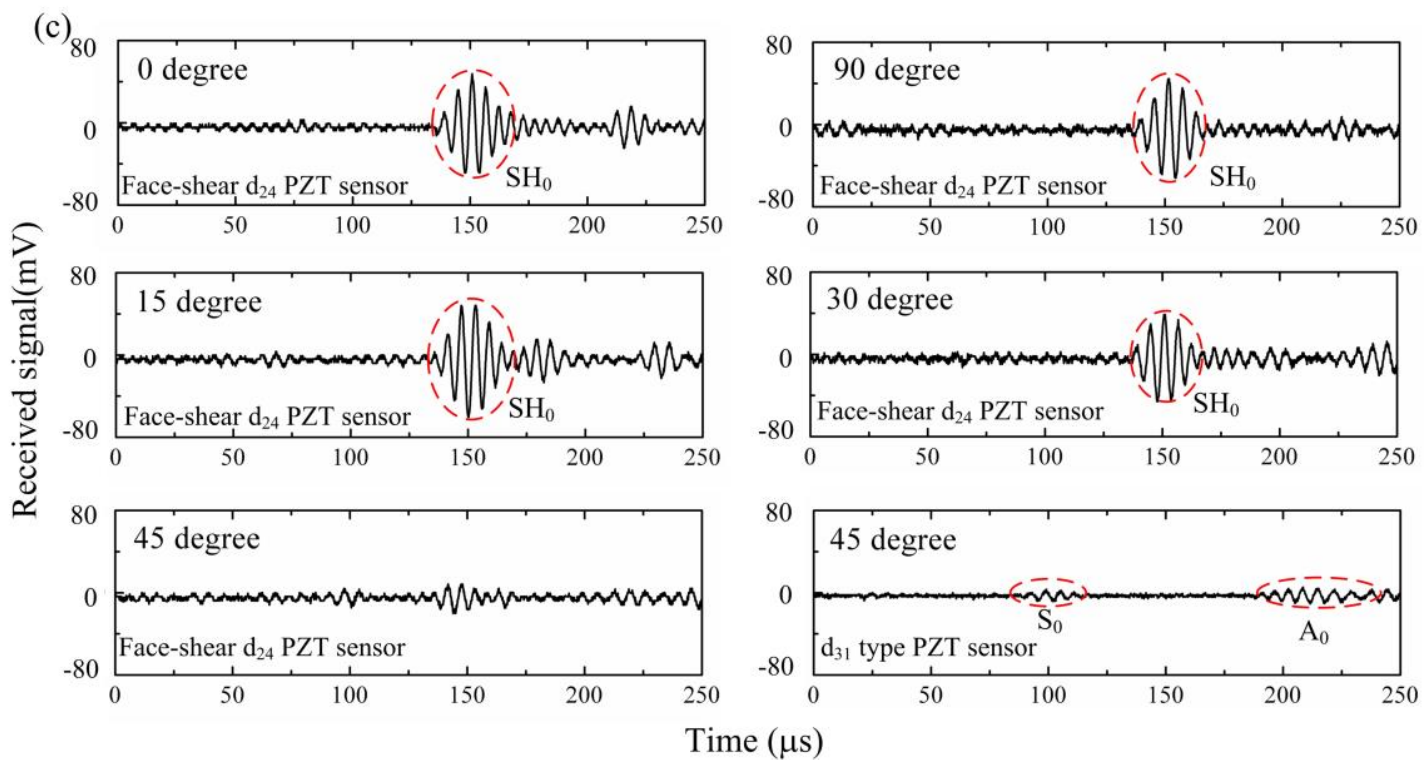

Fig. 6 FEM simulated displacement wavefields excited by the $\mathrm{d}_{24}$ PZT transducer at $150 \mathrm{kHz}$ : (a) 
tangential displacement component ( $\mathrm{SH} 0$ wave) in the cylindrical coordinate, (b) total displacement. (c) Experimental results: SH0 wave received by the face-shear $\mathrm{d}_{24}$ PZT sensor or a $\mathrm{d}_{31}$ PZT sensor at $150 \mathrm{kHz}$ along different propagation directions.

\section{Conclusions}

In summary, we proposed a face-shear $\mathrm{d}_{24}$ PZT wafer to excite and receive pure $\mathrm{SH} 0$ wave in plate structures. Finite element simulations indicate that the face-shear $d_{24}$ transducer is more effective than the thickness-shear $d_{15}$ transducer in driving SH waves. Experimental results show that pure SH0 wave with high signal-to-noise ratio (SNR) can be generated by the face-shear $\mathrm{d}_{24}$ PZT wafer over a wide frequency range. Moreover, the $\mathrm{d}_{24}$ PZT wafer can also serve as a selective SH wave sensor which can exclude lamb waves from $120 \mathrm{kHz}$ to $230 \mathrm{kHz}$. The directionality of the excited SH0 wave was further investigated and it was found that pure SH0 wave can be excited along two orthogonal main directions $\left(0^{\circ}\right.$ and $\left.90^{\circ}\right)$. Furthermore, the excited SH0 wave can keep its amplitude when the deviate angle referring to the main direction $\left(0^{\circ}\right.$ or $\left.90^{\circ}\right)$ is less than $30^{\circ}$. The proposed face-shear $d_{24}$ PZT transducer could be of great importance in the fields of NDT and SHM, since it can excite/receive pure SH wave and be widely used as a cost-effective transducer.

\section{References}

[1] Rose J L 2014 Ultrasonic Guided Waves in Solid Media. (New York:Cambridge University Press).

[2] Ribichini R, Cegla F, Nagy P B and Cawley P 2011 Study and Comparison of Different EMAT Configurations for SH Wave Inspection IEEE T. Ultrason. Ferr 58 2571-2581.

[3] Ma Q Z, Jiao J P, Hu P, Zhong X, Wu B and He C F 2014 Excitation and Detection of Shear Horizontal Waves with Electromagnetic Acoustic Transducers for Nondestructive Testing of Plates Chinese Journal of Mechanical Engineering 27 428-436.

[4] Velichko A and Wilcox P D 2009 Excitation and scattering of guided waves: Relationships between solutions for plates and pipes J. Acoust. Soc. Am. 125 3623-3631.

[5] Thompson R B 1979 Generation of Horizontally Polarized Shear-Waves in Ferromagnetic Materials Using Magnetostrictively Coupled Meander-Coil Electromagnetic Transducers Appl. Phys. Lett. 34 175-177.

[6] Vasile C F and Thompson R B 1979 Excitation of Horizontally Polarized Shear Elastic-Waves by Electromagnetic Transducers with Periodic Permanent-Magnets J. Appl. Phys. 50 2583-2588.

[7] Liu Z H, He C F, Wu B, Wang X Y and Yang S M 2006 Circumferential and longitudinal defect detection using $\mathrm{T}(0,1)$ mode excited by thickness shear mode piezoelectric elements 
Ultrasonics 44 E1135-E1138.

[8] Kamal A and Giurgiutiu V 2014 Shear horizontal wave excitation and reception with shear horizontal piezoelectric wafer active sensor (SH-PWAS) Smart Materials and Structures 23 085019 .

[9] Boivin G, Viens M and Belanger P 2016 Development of a low frequency shear horizontal piezoelectric transducer for the generation of plane SH waves AIP Conference Proceedings 1706 030019 .

[10] Zhou W S, Li H and Yuan F G 2014 Guided wave generation, sensing and damage detection using in-plane shear piezoelectric wafers Smart Materials and Structures 23015014.

[11] Zhou W S, Li H and Yuan F G 2015 Fundamental understanding of wave generation and reception using d(36) type piezoelectric transducers Ultrasonics 57 135-143.

[12] Zhang S J, Jiang W H, Meyer R J, Li F, Luo J and Cao W W 2011 Measurements of face shear properties in relaxor-PbTiO3 single crystals J. Appl. Phys. 110064106.

[13] Miao H C and Li F X 2015 Realization of face-shear piezoelectric coefficient d36 in PZT ceramics via ferroelastic domain engineering Appl. Phys. Lett. 107122902.

[14] Miao H C, Chen X, Cai H R and Li F X 2015 Comparative face-shear piezoelectric properties of soft and hard PZT ceramics J. Appl. Phys. 118214102.

[15] Miao H C, Dong S X and Li F X 2016 Excitation of fundamental shear horizontal wave by using face-shear (d36) piezoelectric ceramics arXiv 1603.05077.

[16] Demma A, Cawley P, Lowe M and Roosenbrand A G 2003 The reflection of the fundamental torsional mode from cracks and notches in pipes J. Acoust. Soc. Am. 114 611-625.

[17] Lowe M J S, Alleyne D N and Cawley P 1998 Defect detection in pipes using guided waves Ultrasonics 36 147-154.

[18] Kwun H and Bartels K A 1996 Experimental observation of elastic-wave dispersion in bounded solids of various configurations J. Acoust. Soc. Am. 99 962-968. 